\title{
Gear-Oil Condition Classification by Means of Support Vector Machine, a first approach
}

Daniel G., Dorigo, Benjamin R., Wiesent, Ana C., Pérez Grassi, Alexander W., Koch:

Technische Universität München, Lehrstuhl für Messsystem- und Sensortechnik, Theresienstr. 90/N5, 80333 München:

+49(0)89 289-23354, d.dorigo@tum.de

\section{Abstract}

During the last years the amount of energy gained from renewable energy sources was steadily increasing and this is still under development. A big role is played by offshore wind parks, which use the wind energy over the see for electricity generation. In order to reduce the high maintaining costs of these plants, different online condition monitoring systems are currently being developed. Especially condition monitoring of gear-oils promises to have an important impact on such maintaining costs. A well-known off-line method for gear-oil quality monitoring is based on the evaluation of their Infrared (IR) spectra.

The analysis of IR spectra, which is performed in specialized laboratories together with other chemical and mechanical tests, is a common tool for lubricant quality evaluation. Important oil parameters affect the IR spectra and can be therefore deduced and classified by spectral analysis. A significant parameter is the Total Acid Number (TAN). The importance of the TAN value lies in its relation with the oil's "age".

In this paper, a preliminary study of gear-oil classification by means of TAN in combination with Support Vector Machines (SVMs) is presented. The Mid-Infrared-spectra (MIR-spectra) of three commercially used gear-oils, a mineral and two synthetic ones, were analyzed and classified according to the range of their TAN value. The classification was performed using up to five classes and tested using full cross validation. The classification results were compared using different kernel functions. The robustness against noise is tested by adding different noise levels to the spectra before classification. This latter leads to a first feasibility check for classifying spectra gained in field application, under rough conditions.

\section{Introduction}

Energy demand is steadily increasing and more and more attention is paid to safeguarding the environment. In this context wind power is one of the most promising green energy sources, especially the one produced in offshore power plants. One problem of these plants is given by their high maintenance costs, from them a considerable part is related to corrective tasks. One way to avoid these corrective tasks is performing a preventive maintenance of the plant. One important preventive action consists in controlling the gear-oil. This control is performed by regularly oil sampling from the wind turbine gear and analyzing it later in specialized laboratories. An established diagnostic method for such analysis is the Fourier-transform-infrared-spectroscopy (FTIR-spectroscopy), which provides relevant information about chemical and lubricating properties of the oil sample. However, one big disadvantage of laboratory analysis is the time delay between sampling and result submission. During this time a problem could cause an unscheduled shutdown.

One alternative to the off-line analysis in laboratories is the implementation of different in-situ measurement systems along the plant. In-situ condition monitoring of important system components such as gear boxes, allows real time detection of random, time discrete events with a reliable registration [1]. This latter enables better scheduling of preventive maintenance actions and contributes to reducing costs and downtime. However, FTIR-spectrometers, such as those used in laboratories, are not best suited for in-situ condition monitoring because of their dimensions and related costs. Miniaturized IR-spectrometers for in-situ applications are already under development [2].

One of the most important parameter for oil quality classification is the Total Acid Number (TAN). This parameter is a measure of the sample acidity [3] and a proxy variable for oil "age". In previous works, the TAN values have been deduced from IR-spectra using Partial Least Squares Regression (PLSR) [4]. However, in many applications, a value prediction is not strictly necessary. A classification of the measured sample in two, three or more groups according to the TAN is fully sufficient.

An established classifier is the Support Vector Machine (SVM). This is a computer algorithm that learns by examples (training) to assign labels to the analyzed objects. This tool finds its application in many different fields like, image [5], financial [6] and spectroscopic data processing [7], [8]. The foundations of SVM have been developed in the late seventies [9], but SVM began to receive a bigger attention in the nineties. The formulation of SVM embodies the Structural Risk Minimization (SRM) principle, which has been shown to be superior to the traditional Empirical Risk Minimization (ERM) principle used by 
conventional neuronal networks. A representative set of oil spectra is used to train the SVM. For a successful training, all considered classes of TAN ranges must be well represented in this set. As the SVM is a supervised classifier, it is necessary to indicate manually to which TAN range belongs each spectrum of the training set. The SVM implementation used in this work is the LIBSVM [10]. For solving multi-class problems, like the one presented in this paper, the LIBSVM uses the "one-against-one" approach [10].

The evaluation of results is performed using the "Leave One Out" (LOO) method (also known as full cross validation). The LOO method consists in training the SVM with all spectra in the training set except for one. The testing is then carried out on the spectrum that was not considered in the training. This procedure is repeated for all spectra in the training set.

\section{Material and Methods}

For the classification task, three commercially available wind turbine gear-oils, a mineral and two synthetic ones (Fig. 1), were analyzed. These three oils were chosen because they are the most used in wind turbines gear-boxes. The synthetic ones are Polyalphaolefin (PAO) oils, where Oil-2 has a higher

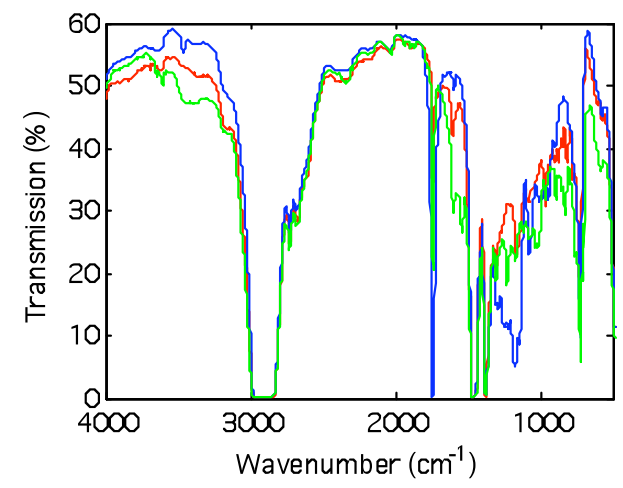

Fig. 1 Transmittance IR-spectra of the analyzed gear-oil types. Oil-1 (red line) is a mineral gear-oil, Oil-2 (blue line) and Oil-3 (green line) are synthetic ones. Oil-2 has a higher ester component than Oil-3.

ester component than Oil-3. Both, the transmittance IR spectra $\mathbf{T}(\bar{v})$ and the absorbance IR spectra $\mathbf{A}(\bar{v})$ will be used for the classification, where $\bar{v}$ denotes the wavenumber given in $\mathrm{cm}^{-1}$. Absorbance spectra are commonly used for spectroscopic data processing. The relation between both spectra is given as follows [11]:

$$
\mathbf{A}(\bar{v})=\log _{10} \frac{1}{\mathbf{T}(\bar{v})}
$$

\subsection{Choosing the best spectral range}

For quantitative analysis of absorbance spectra, the Bouguer-Beer-Lambert law [11], also known as Beer's law, has to be respected. This law states that the component's concentrations in a homogeneous solution is linearly proportional to the intensities of the corresponding absorbance peaks until the absorbance level is below 2.5 (a. u.) [4], [11]. In order to keep the relationship linear, the following regions were excluded for both, the transmittance and the absorbance spectra: $[3067-2758] \mathrm{cm}^{-1},[1500-$ 1330] $\mathrm{cm}^{-1}$.

The spectral region between $\bar{v}=1500 \mathrm{~cm}^{-1}$ and $\bar{v}=500 \mathrm{~cm}^{-1}$ is called "fingerprint region", because their spectral features are characteristic and unique for each sample. In this work, a comparison between using only the fingerprint region and the full spectrum for classification is presented.

\subsection{Selecting classes and SVM parameters}

The complete range of TAN values of interest was subdivided into up to five classes. For the case with two and three classes, the gap between the classes was set to $10 \%$ of the considered TAN value range. For the other case, four and five classes, the gap was set to $5 \%$ of the TAN value range. Tab. 1 shows the range values for each class for the different considered oils. For the presented experiments, each class was represented by spectra with a uniformly distributed TAN. 
Tab. 1 Sub-division of the TAN values into up to five classes for each oil type. The values are given in $\mathrm{mgKOH} / \mathrm{g}$ (milligram potassium hydroxide per oil gram). The gap between the different classes corresponds to $10 \%$ of the considered TAN value range for the case of two and three classes, and to $5 \%$ for the case of four and five classes.

\begin{tabular}{|l||c|c|c|c|c|}
\hline \multicolumn{7}{|c|}{ Oil-1 } \\
\hline \multicolumn{1}{|c||}{} & \multicolumn{7}{c|}{ TAN range $(\mathrm{mgKOH} / \mathrm{g})$} & Class 5 \\
\cline { 2 - 6 } & Class 1 & Class 2 & Class 3 & Class 4 & - \\
\hline 2 Classes & $0.205-0.745$ & $0.755-1.195$ & - & - & - \\
\hline 3 Classes & $0.205-0.462$ & $0.572-0.828$ & $0.938-1.195$ & - & - \\
\hline 4 Classes & $0.178-0.398$ & $0.453-0.673$ & $0.728-0.948$ & $1.003-1.223$ & $1.058-1.223$ \\
\hline 5 Classes & $0.178-0.343$ & $0.398-0.563$ & $0.618-0.783$ & $0.838-1.003$ & \\
\hline
\end{tabular}

\begin{tabular}{|c|c|c|c|c|c|}
\hline \multicolumn{6}{|c|}{ Oil-2 } \\
\hline & \multicolumn{5}{|c|}{ TAN range $(\mathrm{mgKOH} / \mathrm{g})$} \\
\hline & Class 1 & Class 2 & Class 3 & Class 4 & Class 5 \\
\hline 2 Classes & $0.405-0.845$ & $0.955-1.395$ & - & - & - \\
\hline 3 Classes & $0.405-0.662$ & $0.772-1.028$ & $1.138-1.395$ & - & - \\
\hline 4 Classes & $0.378-0.598$ & $0.653-0.873$ & $0.928-1.148$ & $1.203-1.423$ & - \\
\hline 5 Classes & $0.378-0.543$ & $0.898-0.763$ & $0.818-0.983$ & $1.038-1.203$ & $1.258-1.423$ \\
\hline
\end{tabular}

\begin{tabular}{|c||c|c|c|c|c|}
\hline \multicolumn{7}{|c|}{ Oil-3 } \\
\hline \multirow{2}{*}{} & \multicolumn{7}{c|}{ TAN range $(\mathrm{mgKOH} / \mathrm{g})$} \\
\cline { 2 - 6 } & Class 1 & Class 2 & Class 3 & Class 4 & Class 5 \\
\hline 2 Classes & $2.434-3.115$ & $3.285-3.965$ & - & - & - \\
\hline 3 Classes & $2.434-2.832$ & $3.002-3.398$ & $3.568-3.965$ & - & - \\
\hline 4 Classes & $2.393-2.733$ & $2.818-3.158$ & $3.243-3.583$ & $3.668-4.008$ & - \\
\hline 5 Classes & $2.393-2.648$ & $2.733-2.988$ & $3.073-3.328$ & $3.413-3.668$ & $3.753-4.008$ \\
\hline
\end{tabular}

All tests were performed using LIBSVM [10] with six different kernel functions: linear, radial basis (RBF), sigmoid and $2^{\text {nd }}, 3^{\text {rd }}$ and $4^{\text {th }}$ order polynomial function. Before training the spectral values were scaled between -1 and 1 according to [12]. Further scaling values were proved in later tests. The trained models were tested using LOO validation.

In order to define the proper number of spectra per class used by training, the tests were repeated using 30 up to 100 uniformly distributed spectra for each class. Furthermore, for the cases of classifying into two and three classes the number of spectra was increased up to 500 spectra per class. The computation time was also taken into consideration in order to evaluate the classification performance.

Finally, in order to optimize the classification accuracy using the model based on the linear kernel function, the penalty parameter $C$ [ref] was analyzed using the method proposed by [10].

\subsection{Model Robustness against Noise}

After parameter optimization, in order to get the best classification accuracy, the approach robustness against noise was tested. This was performed training the system with the original spectra, which present very low noise, and adding different noise levels on the spectra used for testing the model. We chose to add white noise, which is not correlated to the measured signal. For white noise simulation we added on each spectral point a random value between $\pm 0.5 \%, \pm 1 \%, \pm 1.5 \%, \pm 2 \%, \pm 3 \%, \pm 5 \%$ and $\pm 10 \%$ of the maximal spectral value. While increasing the noise level, the degradation of the prediction rate was analyzed.

\section{Results and Discussion}

As explained in section 3.1, six kernel functions were used for classification of transmittance and absorbance spectra of the three oil types under test. The classification was performed using 50 spectra per class.

As shown in Tab. 2 for transmittance spectra of Oil-1, the classification accuracy decreases clearly with increasing number of classes. The same behavior was noticed for Oil-2 and Oil-3. 
Tab. 2 Classification accuracy for classification in up to five classes using six different kernel functions. 50 full transmittance spectra of Oil-1 per class were used. A decrease in the accuracy related to an increase of classes can be observed.

\begin{tabular}{|c|c|c|c|c|c|c|}
\hline \multicolumn{7}{|c|}{ Oil-1 Transmittance Full Spectra } \\
\hline $\begin{array}{c}\text { Kernel } \\
\text { Function }\end{array}$ & Linear & $\begin{array}{c}2^{\text {nd }} \text { order } \\
\text { polynomial }\end{array}$ & $\begin{array}{c}4^{\text {rd }} \text { order } \\
\text { polynomial }\end{array}$ & $\begin{array}{c}4^{\text {th }} \text { order } \\
\text { polynomial }\end{array}$ & Radial Basis & Sigmoid \\
\hline \hline 2 Classes & 82.0 & 87.0 & 86.0 & 86.0 & 59.0 & 57.0 \\
\hline 3 Classes & 84.0 & 75.3 & 76.0 & 76.0 & 14.0 & 35.3 \\
\hline 4 Classes & 71.0 & 62.5 & 62.5 & 63.0 & 10.5 & 18.5 \\
\hline 5 Classes & 56.0 & 48.8 & 54.0 & 50.8 & 14.4 & 15.2 \\
\hline
\end{tabular}

In Tab. 2 can also be seen that the linear and the $3^{\text {rd }}$ order polynomial kernel function deliver the best classification results. This was also the case for the other two oil types. On the other hand, no considerable difference was noticed between the evaluation of transmittance and absorbance spectra (see Fig. 2).

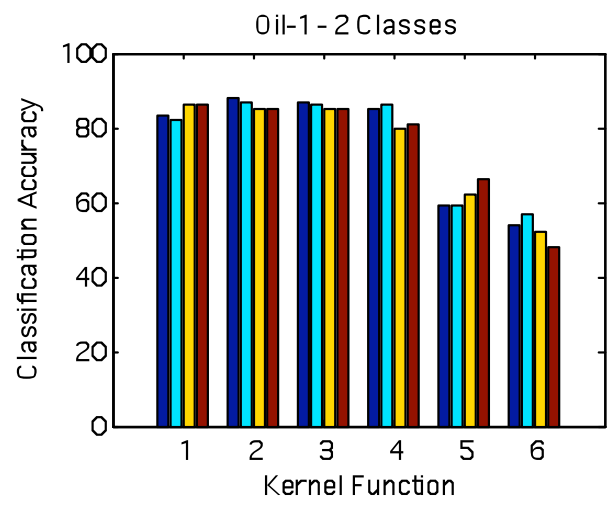

Absorbance Full Spectrum Transmittance Full Spectrum Absorbance Finger Print Transmittance Finger Print
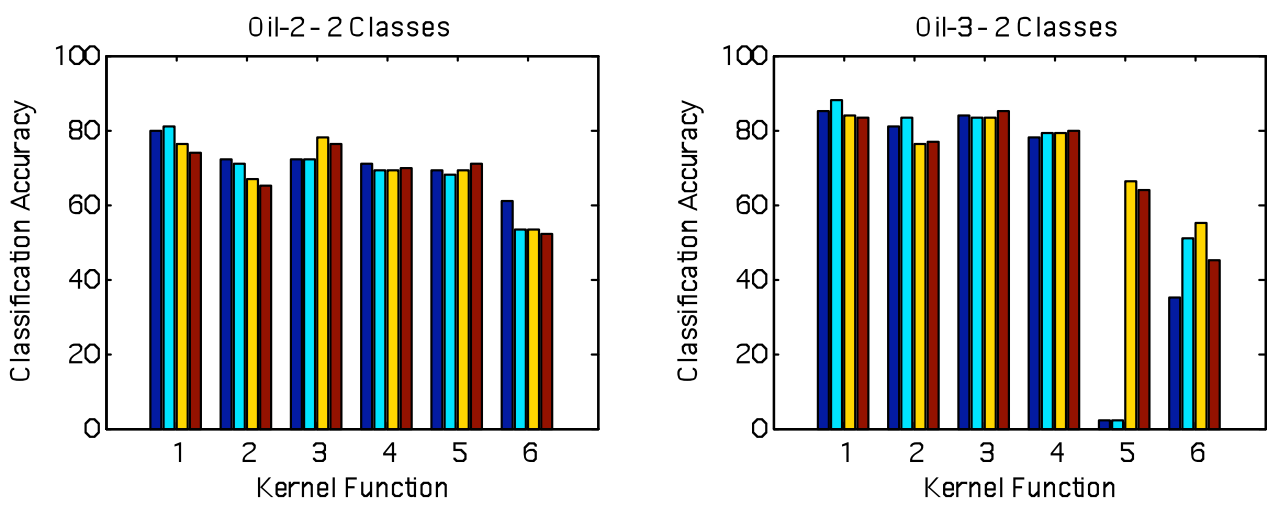

Fig. 2 Classification accuracy for a two-class classification task using different kernel functions. The kernel functions are labelled with the numbers 1 to 6 , which correspond to linear, $2^{\text {nd }}$ order polynomial, $3^{\text {rd }}$ order polynomial, $4^{\text {th }}$ order polynomial, radial basis (RBF) and sigmoid function respectively.

Another point mentioned in section 3.1 is the choice between analyzing the full spectrum against analyzing only the fingerprint region. Fig. 2 shows that, the classification accuracy (two-class classification task) obtained using the full spectral region was better in the most cases. The same behavior was presented for the classification in three-, four- and five-classes too.

Considering the previous results, the following tests were performed using the linear and the $3^{\text {rd }}$ order polynomial kernel function with full transmittance spectra. To test the influence of the number of spectra in the training list, the classification is now performed using up to 500 spectra per class. In Tab. 3, the case of Oil-1 with up to 250 spectra per class can be seen. For a number of spectra higher than 100 , the classification accuracy changed only marginally. Fig. 3 shows the exponentially growing computational time related to the increasing number of spectra for Oil-1 in a two-class classification problem. Oil-2 and 
Oil-3 presented a similar gradient in the computational time, also accompanied by an only marginal increase of the classification accuracy. In order to preserve both, classification rate and computational

Tab. 3 Classification accuracies for Oil-1 using up to 500 spectra per class. The spectra are classified in two to five classes using the linear and the $3^{\text {rd }}$ order polynomial kernel function (poly3).

\begin{tabular}{|c||c|c|c|c|c|c|c|}
\hline \multicolumn{9}{|c|}{ Oil-1 } \\
\hline \multicolumn{10}{|c|}{} & \multicolumn{7}{|c|}{ Classification Accuracy (\%) } \\
\hline No. of Spectra & 40 & 60 & 80 & 100 & 150 & 200 & 250 \\
\hline \hline 2 Classes, linear & 87.5 & 84.2 & 87.5 & 89.5 & 88.7 & 90.8 & 91.8 \\
\hline 3 Classes, linear & 76.7 & 83.9 & 84.6 & 85.0 & 83.6 & 84.3 & 86.3 \\
\hline 4 Classes, linear & 63.8 & 60.4 & 71.6 & 68.3 & - & - & - \\
\hline 5 Classes, linear & 56.5 & 59.0 & 59.5 & 63.0 & - & - & - \\
\hline 2 Classes, poly3 & 80.0 & 84.2 & 81.3 & 85.5 & 86.0 & 87.0 & 87.4 \\
\hline 3 Classes, poly3 & 74.2 & 78.9 & 75.4 & 75.0 & 77.3 & 77.5 & 80.7 \\
\hline 4 Classes, poly3 & 61.9 & 51.7 & 64.4 & 63.8 & - & - & - \\
\hline 5 Classes, poly3 & 48.0 & 51.3 & 49.8 & 53.0 & - & - & - \\
\hline
\end{tabular}

time, we decided to use 100 spectra per class for the next tests. Tab. 5 gives the corresponding classification results using 100 spectra per class for Oil-2 and Oil-3.

Tests for the choice of the best scaling factor [12] were performed for scaling between -3 and 3 , between -5 and 5 and between -10 and 10 using 100 spectra per class. These tests delivered only marginal changes in the accuracy. A change of the scaling margin causes a variation of the weighting of the different kind of features. In our tests, only features of the same type (spectral points) were used. Due to this, there is no significant change in the classification accuracy for data sets with different scaling margin. We decided to carry out our experiment with scaled datasets between the margins -1 and 1 .

An improvement of the classification accuracy can be achieved by optimizing the penalty parameter $C$ of the linear polynomial function, which was set by default to 1 in the previous experiments [10]. Tab. 4 shows the results for all oil types in the case of two and three classes, where an improvement up to $3 \%$ was achieved.
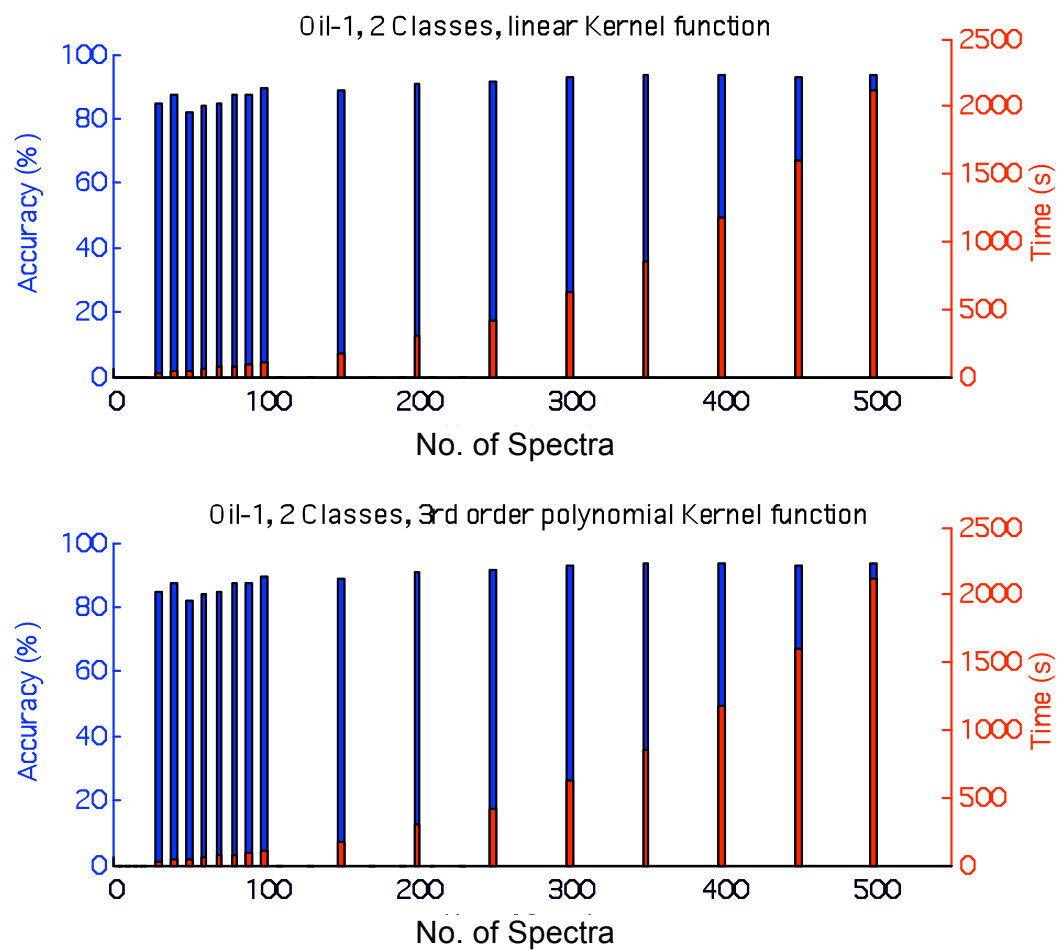

Fig. 3 Relation between accuracy and computational time for a two-class classification task (Oil-1). The linear and the $3^{\text {rd }}$ order polynomial kernel function were used. An exponential rise in the computational time with increasing number of spectra per class can be seen. 
Tab. 5 Classification accuracy for Oil-2 and Oil-3 using 100 spectra per class. The spectra are classified in two to five classes using the linear and the $3^{\text {rd }}$ order polynomial kernel function (poly3).

\begin{tabular}{|c|c|c|}
\hline & Oil-2 & Oil-3 \\
\cline { 2 - 3 } & Classification & Accuracy (\%) \\
\hline No. of Spectra & 100 & 100 \\
\hline \hline 2 Classes, linear & 89.5 & 87.0 \\
\hline 3 Classes, linear & 81.3 & 80.3 \\
\hline 4 Classes, linear & 75.8 & 73.8 \\
\hline 5 Classes, linear & $72.3^{*}$ & 63.6 \\
\hline 2 Classes, poly3 & 81.5 & 86.0 \\
\hline 3 Classes, poly3 & 81.3 & 77.3 \\
\hline 4 Classes, poly3 & 72.8 & 67.3 \\
\hline 5 Classes, poly3 & $64.4^{*}$ & 60.0 \\
\hline
\end{tabular}

${ }^{*}$ The $5^{\text {th }}$ class contains 94 spectra instead of 100 .

The approach robustness against noise (see section 3.3) was tested on the data sets without optimization of the penalty parameter $(C=1)$. As it can be seen in Fig. 4, for Oil-1 the accuracy decreases exponentially with the noise level until a saturation level is reached. The approach based on the $3^{\text {rd }}$ order polynomial kernel function shows a better robustness compared to that based on the linear kernel

Tab. 4 Optimization of the penalty parameter C for the SVM model based on the linear kernel function.

\begin{tabular}{|c|c||c|c|c|}
\hline \multicolumn{2}{|c|}{} & c & Accuracy (\%) & Improvement (\%) \\
\hline \hline \multirow{2}{*}{ Oil-1 } & 2 Classes & 1.5 & 91.0 & 1.5 \\
\cline { 2 - 5 } & 3 Classes & 0.5 & 87.0 & 2.0 \\
\hline \multirow{2}{*}{ Oil-2 } & 2 Classes & 19.1 & 91.5 & 2.0 \\
\cline { 2 - 5 } & 3 Classes & 0.4 & 84.7 & 3.3 \\
\hline \multirow{2}{*}{ Oil-3 } & 2 Classes & 1.2 & 88.0 & 1.0 \\
\cline { 2 - 5 } & 3 Classes & 0.14975 & 83.7 & 3.3 \\
\hline
\end{tabular}

function. However, the last shows a better classification accuracy for low noise levels. For the case of the $3^{\text {rd }}$ order polynomial kernel function used in a two-class classification task, the performed classification is acceptable until a noise level of $3 \%$. A similar characteristic is shown by the other two oil types too. All results for the two-class classification task can be seen in Tab. 6.

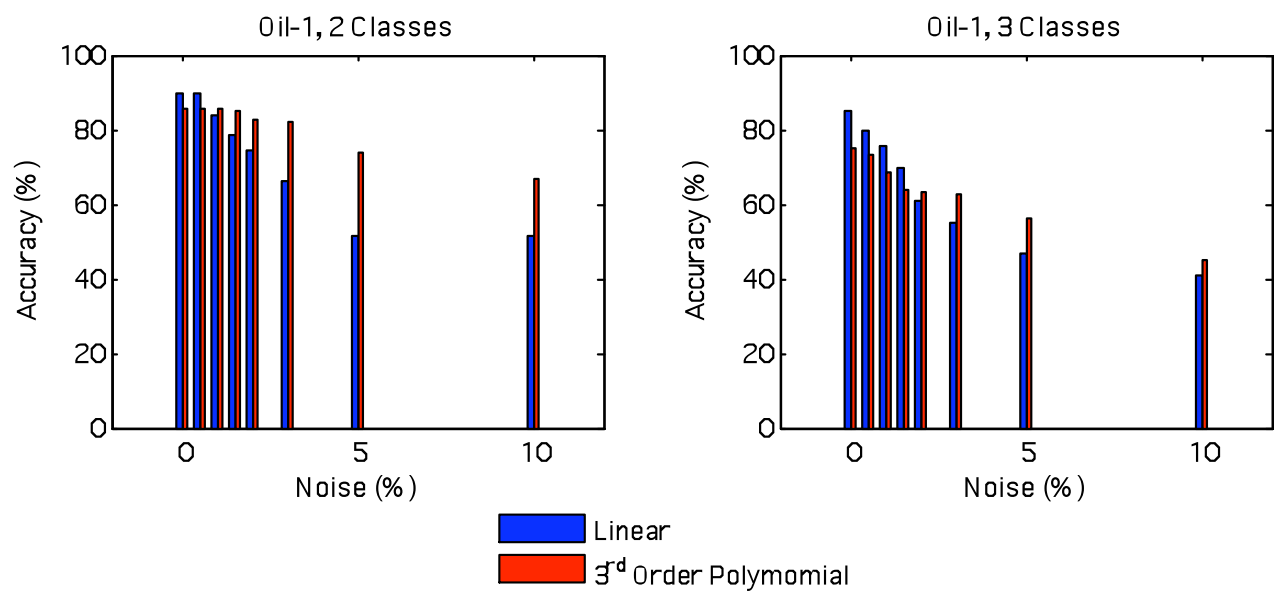

Fig. 4 Classification accuracy for a two- and three-class classification task of noisy spectra using the linear and the $3^{\text {rd }}$ order polynomial kernel function. Different noise levels were added to the spectra before testing the model. The model based on the $3^{\text {rd }}$ order polynomial kernel function presents a higher robustness against noise. 
Tab. 6 Classification accuracy for a two- class classification task of noisy spectra using the linear and the $3^{\text {rd }}$ order polynomial (poly3) kernel function.

\begin{tabular}{|c|r|r|r|r|r|r|}
\hline & \multicolumn{4}{|c|}{ Classification Accuracy (\%) } \\
\cline { 2 - 8 } & \multicolumn{2}{|c|}{ Oil-1 } & \multicolumn{2}{c|}{ Oil-2 } & \multicolumn{2}{c|}{ Oil-3 } \\
\hline Kernel Function & Linear & \multicolumn{1}{c|}{ Poly3 } & \multicolumn{1}{c|}{ Linear } & \multicolumn{1}{c|}{ Poly3 } & \multicolumn{1}{c|}{ Linear } & Poly3 \\
\hline \hline $0.0 \%$ noise & 89.5 & 85.5 & 89.5 & 81.5 & 87.0 & 86.0 \\
\hline $0.5 \%$ noise & 89.5 & 85.5 & 80.0 & 79.0 & 81.5 & 85.0 \\
\hline $1.0 \%$ noise & 84.0 & 85.5 & 76.5 & 71.0 & 77.0 & 78.0 \\
\hline $1.5 \%$ noise & 78.5 & 85.0 & 71.0 & 67.0 & 73.0 & 77.0 \\
\hline $2.0 \%$ noise & 74.5 & 82.5 & 64.5 & 63.5 & 73.0 & 73.5 \\
\hline $3.0 \%$ noise & 66.0 & 82.0 & 56.0 & 56.5 & 66.5 & 67.0 \\
\hline $5.0 \%$ noise & 51.5 & 74.0 & 56.0 & 55.0 & 56.0 & 60.0 \\
\hline $10.0 \%$ noise & 51.5 & 67.0 & 55.5 & 59.5 & 60.0 & 54.5 \\
\hline
\end{tabular}

\section{Conclusions}

In this work, the use of SVMs as classifier for oil spectra according to the range of their TAN value was tested. The spectra of three typical commercially available gear oils were subdivided into maximal five classes according to their TAN value and classified using different kernel functions and SVM parameters. Classification results using transmittance and absorbance spectra were compared. No relevant differences in the classification accuracy could be noticed. The use of the complete spectrum as feature for the classificatory was also compared with the use of the fingerprint region, where the first one gave the best results. Different kernel functions were tested, where the linear and $3^{\text {rd }}$ order polynomial functions gave the best results.

Increasing the number of considering spectra per classes during the training, a slight improvement of the classification rate could be observed. However, together with the number of spectra, the computational time increases exponentially, which requires a compromise solution.

An optimization of the penalty parameter delivers an improvement in the results. In this work only the optimization for the linear kernel function was performed. Extending this optimization also to other kernel functions could result in a stronger impact in the classification accuracy, due to the fact that more parameters are involved.

The linear kernel function showed the best classification accuracies, however, the $3^{\text {rd }}$ order polynomial function turned out to be the most robust against noise influence.

Concluding, this work showed that SVM is a promising tool for classifying oils spectra according to the range of their TAN values. This could be used to set alarms on an in-situ monitoring system, which indicates if a gear-oil is inside acceptable values or not. This work used the complete oil's spectra as feature for the classification without preprocessing; in a future work the potency of other features extracted from the spectra will be studied.

\section{Acknowledgements}

The authors would like to thank Oelcheck $\mathrm{GmbH}$ for providing the oil samples and the correspondent analysis reports. Funding by "Bayerisches Statsministerium für Wirtschaft, Verkehr und Technologie" and administrative support of VDI/VDE are gratefully acknowledged. This work was also supported by the TUM Graduate School.

\section{References}

[1] R. Krethe, "Oil Condition Monitoring - Online, Onsite or Lab analysis?," in Proceedings of OilDoc, 2011.

[2] B. R. Wiesent, D. G. Dorigo, Ö. Simsek, and A. W. Koch, "Linear variable filter based oil condition monitoring systems for offshore windturbines," in Proceedings of SPIE, 2011, vol. 8105, no. 81050, p. 81050D.

[3] D. M. Pirro, A. A. Wessol, and J. G. Wills, Lubrication Fundamentals, Second Edi. CRC, 2001.

[4] B. R. Wiesent, D. G. Dorigo, M. Schardt, and A. W. Koch, "Gear oil condition monitoring for offshore wind turbines using band limited low resolution spectra," Proceedings of Oildoc, 2011. 
[5] A. Pérez Grassi and F. Puente León, "Invariante Merkmale zur Klassifikation von Defekten aus Beleuchtungsserien (Invariant Features for Classification of Defects Based on Illumination Series)," tm - Technisches Messen, vol. 75, no. 7-8, pp. 455-463, Jul. 2008.

[6] A. Fan and M. Palaniswami, "Selecting bankruptcy predictors using a support vector machine approach," Proceedings of the IEEE-INNS-ENNS International Joint Conference on Neural Networks. IJCNN 2000. Neural Computing: New Challenges and Perspectives for the New Millennium, no. 2, pp. 354-359 vol.6, 2000.

[7] K. M. Almhdi, P. Valigi, V. Gulbinas, R. Westphal, and R. Reuter, "Classification with Artificial Neural Networks and Support Vector Machines: application to oil fluorescence spectra," EARSeL eProceedings, vol. 6, no. 2, pp. 115-129, 2007.

[8] O. Devos, C. Ruckebusch, A. Durand, L. Duponchel, and J.-P. Huvenne, "Support vector machines (SVM) in near infrared (NIR) spectroscopy: Focus on parameters optimization and model interpretation," Chemometrics and Intelligent Laboratory Systems, vol. 96, no. 1, pp. 27-33, Mar. 2009.

[9] V. Vapnik, Estimation of dependences based on empirical data (in Russian). Nauka, Moskow. English translation 1982 Spriger Verlag, New York, 1979.

[10] C. C. Chang and C. J. Lin, "LIBSVM: a library for support vector machines," ACM Transactions on Intelligent Systems and Technology (TIST), vol. 2, no. 3, p. 27, 2011.

[11] F. A. Settle, Ed., "Infrared Spectroscopy," in Handbook of Instrumental Techniques for Analytical Chemistry, Prentice Hall PTR, 1997, pp. 247-284.

[12] C. W. Hsu, C. C. Chang, C. J. Lin, and others, "A practical guide to support vector classification," Bioinformatics, vol. 1, no. 1. pp. 1-16, 2003. 